\title{
SOUND AND VIBRATION CONSIDERATIONS OF SOME MATERIALS FOR AUTOMOTIVE ENGINEERING APPLICATIONS
}

\author{
${ }^{1}$ Mohammad Al-Zubi, ${ }^{2}$ Emmanuel Ayorinde, \\ ${ }^{1}$ Nabeel Alshabatat, ${ }^{2}$ Mehmet Dundar and ${ }^{3}$ Yellapu Murty \\ ${ }^{1}$ Department of Mechanical Engineering, Tafila Technical University, Jordan \\ ${ }^{2}$ Department of Mechanical Engineering, Wayne State University, Detroit, USA \\ ${ }^{3}$ MC Technologies, 1163 River Chase Ridge, Charlottesville, VA 22901, USA
}

Received 2014-02-23; Revised 2014-05-08; Accepted 2014-10-27

\begin{abstract}
Vibration and noise amelioration in and across several components and modules of the automotive, such as the panels, doors, engine covers, seats and others, is extremely important. NVH performance has been recognized as a critical factor in the purchase decisions of many buyers. This study examines the vibro-acoustic characteristics of some monolithic and composite materials and some generally periodic material structures. By experimental and numerical/empirical methods, it explores some opportunities for minimizing the transmission of noise and vibration. Some new constructions not hitherto probed for vibro-acoustic fitness are examined. Results from this study are expected to contribute towards design inputs to obtain better performances.
\end{abstract}

Keywords: Noise, Vibration, NVH, Composite, Vibro-Acoustic, Automotive

\section{INTRODUCTION}

Although automobiles, marine craft and aeronautical structures all need good vibro-acoustics, the first case is probably more critical because there are far many more automobiles than ships or aircraft. The qualities of importance initially advertized by automotive vehicle manufacturers were performances in terms of horsepower and speed. Historically, consumers demanded progressively more driver and passenger comfort satisfaction for their vehicles. This induced the input of significant levels of effort by manufacturers into the systematic reduction, containment and possible elimination of noise and vibration in the automotive environment. In this pursuit, the preliminary measure was that of simply stuffing as much insulation as practicable into the panels (door, roof, floor) to curb rattling movements and produce quiet. It has been recalled by Saha
(Vardan, 2003) that the earlier power-trains were so noisy that the wind noise could not even be heard at all from within the vehicle, having been overshadowed by the internal vehicle noise. The power train noise was soon conquered, but concern over wind noise immediately surfaced. He remarked from historical data that the persistent pursuit of ever increasing noise and vibration performance has been driven mainly by customer demand and not legal or regulatory requirements and standards.

Kropp (Vardan, 2003) concluded that "noise and vibration have become a statement of car quality" and further stated that people buy cars to get from one point to another-reliably and comfortably, but also expect a quiet ride for their money and usually want, among other things, to listen to the car radio without disturbance by much noise. This view from academic experts in the automotive noise and vibration field expresses the mind of automotive industry experts as Corresponding Author: Ayorinde E., Department of Mechanical Engineering, Wayne State University, Detroit, MI 48202, USA 
well. Parry Jones (Parry-Jones and Weaver, 1999), Group Vice President, Ford Motor Co. said "NVH is overwhelmingly important to customers. You never, ever get lucky with NVH. The difference between good cars and great cars is fanatical attention to detail".

Noise is the term used for unwanted sound; vibration means to-and-fro motion; and harshness is the term often used for subjective perception of low-frequency (25-100 $\mathrm{Hz}$ ) vehicle vibration, usually perceived as rough, grating and discordant. Noise, Vibration and Harshness (NVH). Is the term often used for the science and technology of managing vibration and noise frequencies, levels, energies and patterns.

The traditional method for constructing the automotive panel structure has been typically to layer a metal panel outer member with a visco-elastic damping layer, then a porous layer and then a rubber/plastic layer in order to improve vibro-acoustic performance. More recently, (Ho and Berkhoff, 2014, Jain, 2002), efforts have been directed in the industry towards improving the nature and architecture of the panel itself, such that extra material and labor costs are saved and yet such panels have good vibroacoustic performance. Exhaustive treatment of vibroacoustics for automotive panels is sparse in the literature and thus still has room for more work.

Accordingly, the objectives of this study are to investigate by experimental, analytical and numerical methods the vibration and acoustic performances of many trial panel materials and to consider what physical and material properties and architectural constructions yield better vibration and acoustic performances and thus advance towards the development of better and newer automotive panels with good NVH performances. The class of Periodic Cellular Material Structures (PCMS) has been investigated by the authors (Al-Zubi et al., 2013), but this article treats several other categories of materials.

Automotive panels and other components come in various shapes. However, for sample test purposes, as long as the same investigative test samples geometry and boundary conditions are used, the comparisons between different materials or constructions remain valid with scaled-up production parts. It is also customary to test a relatively few number of full-scale parts. Thus it has been found satisfactory to test large numbers of relatively simple (such as circular, rectangular,) parts, to start with. The scientific aspects of the work center on sound and vibration and the primary considerations are those of the transmission of sound through materials and also the vibration of plates and plate-like materials.

With respect plate vibration, Poisson (1894) first examined the vibrations of circular plates, analyzing the fixed, simply supported and free edges cases. Kirchhoff (1850) extended Poissons work to more cases, giving the full theory for the free circular plate case. Rayleigh (1945) developed a general theory for all cases of vibrating circular plates, based on an energy principle. Timoshenko (2011) analyzed the transverse vibration of variously-constrained circular and rectangular plates and obtained natural frequency formulas. Hoshino et al. (2003) simulated and analyzed the vibration reduction of heavy duty truck cabins. Wang et al. (2009) investigated plate vibration mode shapes and concluded that the mode- 2 mode shape may be identical to mode- 1 except for a $45^{\circ}$ rotation and the resulting mode- 3 may be identical to the standard mode-2 (i.e., having one nodal circle).

Concerning acoustics, Zwikker and Kosten (1949) gave a deep literature review with data and tables on the sound absorption coefficients of several materials. Kurtze (1959) developed a principle of wave propagation. Dym and Lang (1974) obtained analytic expressions for impedance and transmission loss of a sandwich panel. Woodcock and Nicolas (1995), for the first time, studied many aspects of low-frequency range sound, with emphasis on the finite-size panel. Wang et al. (2010) combined various numerical calculation methods to model and analyze the acoustic characteristics of a heavy truck cab.

Many materials could be deployed in the effort to reduce sound and vibration. Fabric-like materials could be highly efficient acoustic absorbers, but in terms of vibration, they would usually be framed in a rigid structure or sandwiched in such layers, so that the assembly could resist vibration. Vibro-acoustic sandwich constructions could be assembled in a wide variety of ways. Basalt and resonated cotton have been known and produced commercially for acoustic treatments (Ross, 2006; ASI, 2012). In this study, for comparison purposes, we tested basalt wool, resinated cotton, glass wool and Polyethylene Terephthalate (PET).

Foam materials, both plastic and metallic, have also been utilized for vibro-acoustic padding purposes. A sandwich construction Comprising Cold Rolled Steel (CRS) skins and a closed-cell, hard foam (LE 5208) core is also examined in this study. 
In a similar way, honeycomb periodic structures are well known and have been put to many uses, although in terms of particular geometries and component materials (skin and core), there are still many new possibilities. It has been noted that traditional honeycomb materials, by their fixed architecture, seriously limit design options, are very difficult if not impossible to make multifunctional and have closed cells, preventing embedment.

\section{THEORY}

\subsection{Acoustics}

The acoustics theory of waves incident on plates has been treated by many authors in books and technical papers. We have followed the development by (Zwikker and Kosten, 1949; Biot, 1956; Allard et al., 1999). The fundamental theories are available in relevant texts and only very brief summaries are relevant here. The acoustic analysis can be established by starting from the Equations of motion and continuity of an infinitesimal layer, thickness $\mathrm{dx}$, of the vibrating medium, under pressure $\mathrm{p}$, as shown in the following figure, neglecting damping effects for simplicity.

By carrying out a force-balance on the acoustic element of Fig. 1, the governing Equation, where $\mathrm{K}$ is bulk modulus, subscript o refers to air, $\rho$ is density and $\omega$ is frequency, becomes Equation 1:

$$
\frac{\partial^{2} P}{\partial x^{2}} \frac{\rho_{0}}{K_{0}} \frac{\partial^{2} P}{\partial t^{2}}
$$

Results, having solution of pattern Equation 2:

$$
p=A \exp (j \omega t) \exp \left(-\gamma_{0} x\right)
$$

And, if $\mathrm{z}$ is plate impedance and $\mathrm{c}_{0}$ is velocity of propagation of sound waves in free air, the acoustic absorption coefficient is finally given by Equation 3:

$$
a_{0}=\frac{4 \operatorname{Re}\left(z / \rho_{0} c_{0}\right)}{\left(\operatorname{Re}\left(z / \rho_{0} c_{0}\right)+1\right)^{2}+\left(\operatorname{Im}\left(z / \rho_{0} c_{0}\right)\right)^{2}}
$$

\subsection{Vibration}

Similarly, we may briefly outline the vibration dynamics of the circular plate, which can be developed in many ways. One way is to use the classical small-deflection theory of plates, developed by Lagrange (1815).
For the circular plate, whose elements are shown in Fig. 2, the potential and kinetic energies, $U$ and $T$ respectively, may be stated as Equation 4 and 5:

$U=\frac{D}{2} \int_{0}^{2 \pi} \int_{0}^{R}\left\{\begin{array}{l}\left(\frac{\partial^{2} w}{\partial r^{2}}+\frac{1}{r} \frac{\partial w}{\partial r}+\frac{1}{r^{2}} \frac{\partial^{2} w^{2}}{\partial \theta^{2}}\right)^{2}-2(1-V) \frac{\partial^{2} w}{\partial r^{2}} \\ \left(\frac{1}{r} \frac{\partial w}{\partial r}+\frac{\partial^{2} w}{\partial \theta^{2}}\right)+2(1-V)\left\{\frac{\partial}{\partial r}\left(\frac{1}{r} \frac{\partial w}{\partial \theta}\right)^{2}\right\}\end{array}\right\} r d \theta d r$

And:

$$
T=\frac{\rho h}{2} \int_{0}^{2 \pi} \int_{0}^{R} \dot{w}^{2} r d r d \theta
$$

Where:

$R=$ Radius at general point, $\mathrm{R}$ is radius of the plate

$w=$ Deflection of the plate

$h=$ The thickness of the plate

$\rho=$ The mass density of the plate

$v=$ The Poisson ratio and E is Young"s modulus of the plate material

$D=$ The flexural (i.e., bending) rigidity of the plate, given by Equation 6 :

$D=\frac{E h^{3}}{12\left(1-v^{2}\right)}$

For harmonic vibrations in axi-symmetric cases, the vibration frequency in all cases can be determined (Timoshenko, 2011) by the Equation 7:

$$
\omega=\frac{\alpha}{R^{2}} \sqrt{\frac{D}{\rho h}}
$$

where, $\alpha$ is called modal constant and D is the modulus of rigidity. For a free circular plate with $\mathrm{n}$ nodal diameters and $s$ nodal circles, the values of $\alpha$ are given for some modes in the following table. Table 1 shows values computed for the first three modes for a steel plate with Poisson"s ratio of 0.33 . Our work showed that Rayleigh"s approximate energy method under-predicted the natural frequency by only $6 \%$ relative to the bessel function solution.

Good automotive NVH performance depends on proper selection of materials and structures that can make maximal contributions to the desired qualities. Effort is therefore being continually made by the automotive companies in the improvement of such materials and structures and methods for their analyses and production. 


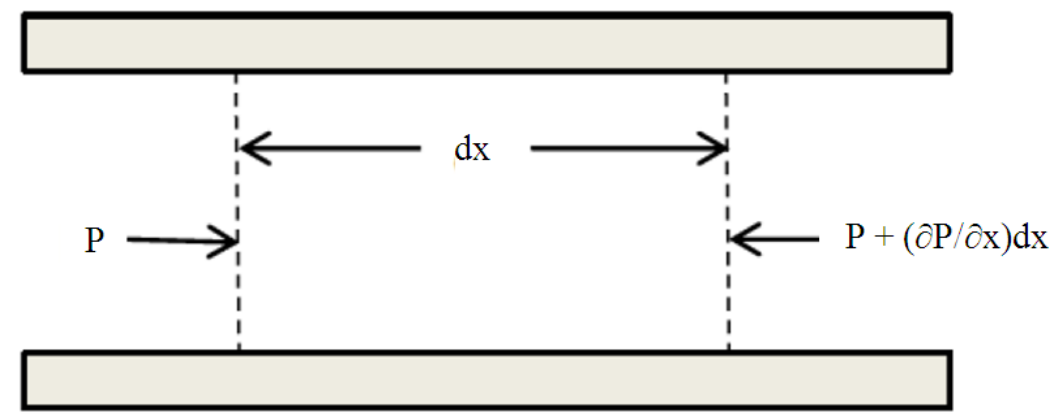

Fig. 1. Acoustic element

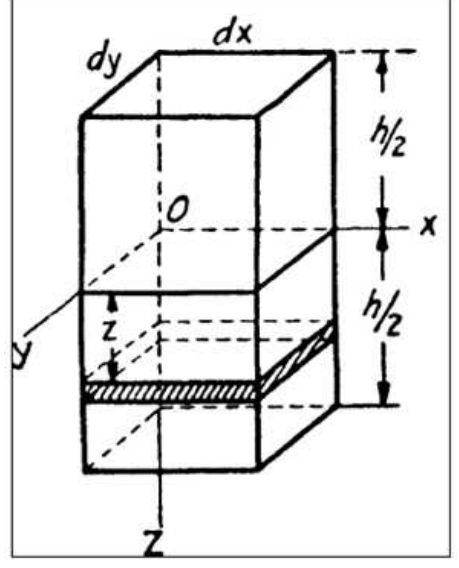

(a)

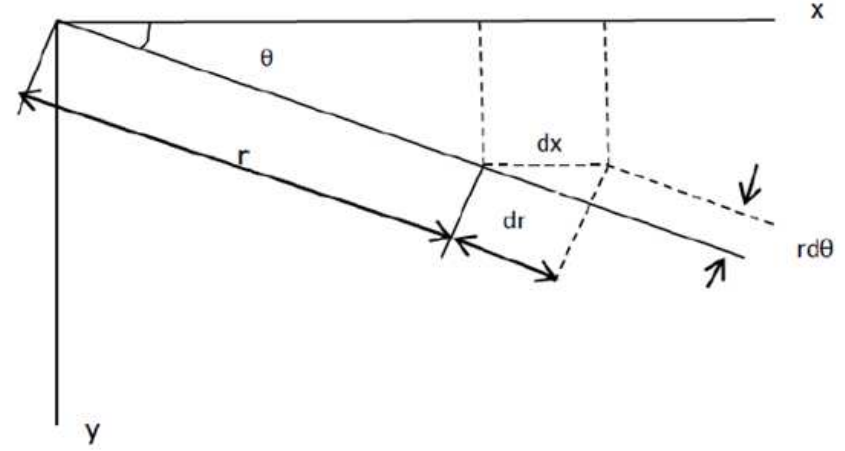

(b)

Fig. 2. (a) Element of vibrating plate, (b) Model of deformed coordinates in plate vibration (Timoshenko, 2011)

Table 1. Sample alpha values for different nodal lines and circles (Timoshenko, 2011)

\begin{tabular}{|c|c|c|c|c|}
\hline \multirow[b]{2}{*}{$\mathrm{S}$} & \multicolumn{4}{|l|}{$\mathrm{n}$} \\
\hline & 0 & 1 & 2 & 3 \\
\hline 0 & ……… & $\ldots \ldots \ldots \ldots \ldots$ & 5.251 & 12.23 \\
\hline 1 & 9.076 & 20.52 & 35.24 & 52.91 \\
\hline 2 & 38.52 & 59.86 & ........ & ........... \\
\hline
\end{tabular}

\section{MATERIALS}

This study examines the acoustic and vibration performances of some fibrous, foam, honeycomb, monolithic and sandwich and materials that could be deployed in automotive panels and general engineering structural components. The study focuses on standard acoustic and vibration performances in terms of acoustic absorption coefficient and vibration frequency response and deflections. The materials tested are shown in Fig. 3. The fibrous materials considered for acoustic absorption are fiberglass, resinated cotton and basalt wool. A thicker, multi-layer fiberglass material and a PET sample were obtained from $\mathrm{B}$ and $\mathrm{K}$ for comparison. Tested foam materials include a wave-formed polyurethane material from $\mathrm{B}$ and $\mathrm{K}$ and two custom-made materials from Athena Engineers, Lake Orion, USA. These were development-stage compounds from a collaborator of theirs, which we expanded in our own laboratories. The seven honeycomb materials tested are listed in Table 2. These include a traditional all-aluminum honeycomb periodic material and a new one with CRS-skin and nomex core. Monolithic lexan plates of various layering and perforation styles and a sandwich material having 
Cold Rolled Steel (CRS) skins and a hard-foam core are among those considered.

The material of the aluminum samples is aluminum 6061 T6 and the steel is stainless steel 304. To the authors" best knowledge, the vibro-acoustics of the new materials have not been considered before.

\section{EXPERIMENTAL SETUP}

The "PULSE" 14.0 (and later the 16, with the software Pulse Reflex 16.1) vibro-acoustic instrumentation and software platform was utilized in our experiments, along with the impedance tube, impact hammer and accelerometers, all from Bruel and Kjaer,
Inc. The tube utilized was the B and $\mathrm{K}$ Two-Microphone Impedance Measurement Tube Type 4206, fitted with two specially designed $1 / 4$-inch microphones. Specimens of diameters $100 \mathrm{~mm}$ and $29 \mathrm{~mm}$ respectively were cut from each material for acoustic absorption tests and the larger samples were also subjected to impact response vibration tests. Results were obtained from experimental, analytical and numerical approaches. Figure $\mathbf{4}$ is an image illustration of essential parts of the acoustic setup.

For the Frequency Response (FRF) test, an impact hammer arrangement is utilized as detailed in Al-Zubi et al. (2013). For the full modal test, we used 17 roving hammer points.
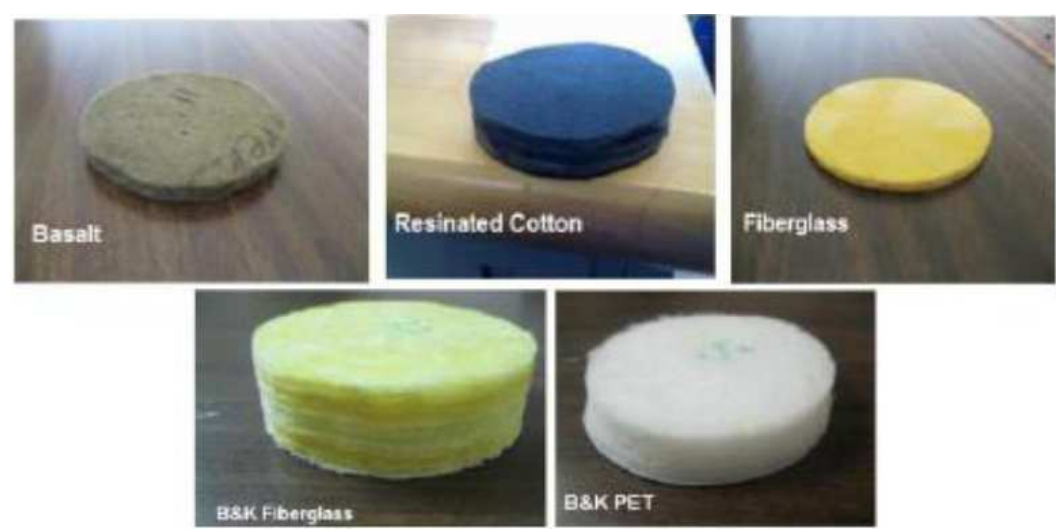

(a)
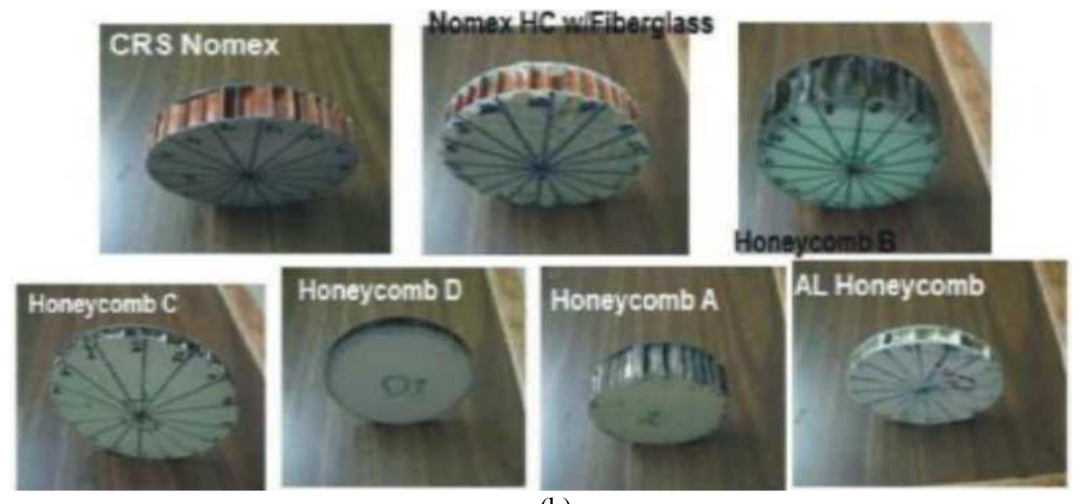

(b)
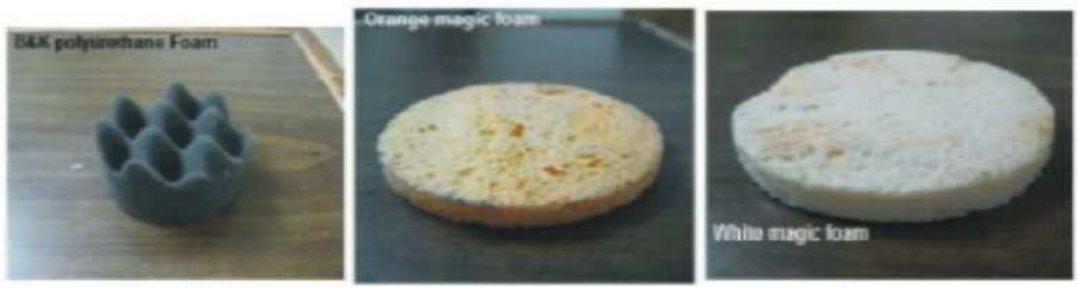

(c) 

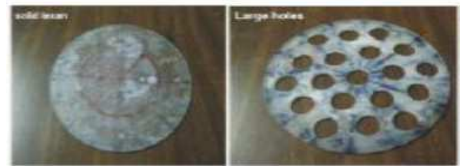

(d)
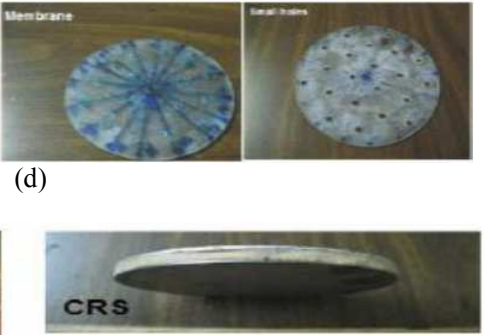

(e)

Fig. 3. (a)-(e). Images of Test Materials (a) Fabric-like materials (b) honeycomb materials (c) foam materials (d) monolithic structures (e) sandwich materials

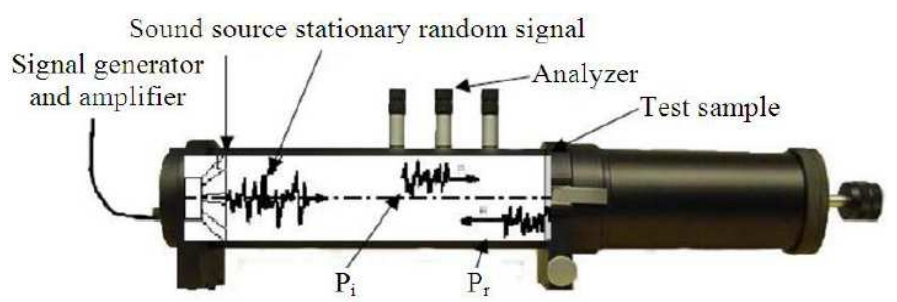

Fig. 4. Sketch of the 2-Microphone impedance method (B and K)

Table 2. Table of tested materials and some properties

(a) Fabrics

\begin{tabular}{lccc}
\hline Material & Weight $(\mathrm{gm})$ & Thickness $(\mathrm{mm})$ & Density $\left(\mathrm{kg} / \mathrm{m}^{-3}\right)$ \\
\hline Basalt & 15.70 & 12.7 & 157.00 \\
Resinated cotton & 24.68 & 17.0 & 184.84 \\
Fiberglass & 4.05 & 9.5 & 54.36 \\
B and K Fiberglass (yellow) & 22.35 & 27.0 & 105.40 \\
B and K PET (poly-ethylene-terephthalate) & 3.91 & 18.0 & 27.65 \\
\hline
\end{tabular}

(b) Foams

\begin{tabular}{lccc}
\hline Material & Weight $(\mathrm{gm})$ & Thickness $(\mathrm{mm})$ & Density $\left(\mathrm{kg} / \mathrm{m}^{-3}\right)$ \\
\hline B and K Polystyrene foam & 7.35 & 32.0 & 29.24 \\
Orange magic foam & 11.47 & 7.5 & 194.72 \\
White magic foam & 24.64 & 11.0 & 285.20 \\
\hline
\end{tabular}

(c) Honeycombs

\begin{tabular}{|c|c|c|c|c|c|c|c|c|}
\hline \# & Panel ID & Skin & Core & $\begin{array}{l}\text { Cell } \\
\text { size (mm) }\end{array}$ & $\begin{array}{l}\text { Weight } \\
(\mathrm{gm})\end{array}$ & $\begin{array}{l}\text { Total density } \\
\left(\mathrm{kg} / \mathrm{m}^{-3}\right)\end{array}$ & $\begin{array}{l}\text { Young"s } \\
\text { modulus }\end{array}$ & $\begin{array}{l}\text { Poisson"s } \\
\text { (MPa) ratio }\end{array}$ \\
\hline 1 & AA.2-95 (A) & $\begin{array}{l}\text { Glass epoxy } \\
\mathrm{t}=0.018^{\prime \prime}\end{array}$ & $\begin{array}{l}\text { Aluminum } \\
\text { Honeycomb } \\
\rho=5.2 \mathrm{pcf}\end{array}$ & 6.3 & 28.34 & 48.0 & 2438 & 0.4 \\
\hline 2 & PP5.0-90 (B) & $\begin{array}{l}\text { Glass epoxy w/ } \\
\text { peel ply t-0.014" }\end{array}$ & $\begin{array}{l}\text { Polypropylene } \\
\text { Honeycomb } \\
\rho=5 \mathrm{pcf}\end{array}$ & 6.3 & 29.5 & 47.0 & 1100 & 0.28 \\
\hline 3 & AA3.6-80 (C) & $\begin{array}{l}\text { Aluminum epoxy } \\
\text { primer finish }(t=0.02)\end{array}$ & $\begin{array}{l}\text { Aluminum } \\
\text { Honeycomb } \\
\rho=3.6 \mathrm{pcf}\end{array}$ & 10.3 & 26.1 & 140.0 & 2100 & 0.35 \\
\hline 4 & PN1-1/8-3.0 (D) & Polyester & Aramid nomex & 6.3 & 17.5 & 89.50 & 2250 & 0.25 \\
\hline 5 & $\mathrm{AL}$ & Aluminum $0.032 ", 0.02 "$ & Aluminum & 10.3 & 40.8 & 130.65 & 2000 & 0.40 \\
\hline 6 & FGN & Fiberglass (0.01") & Nomex HC & 6.3 & 21.0 & 52.86 & 1800 & 0.37 \\
\hline 7 & $\mathrm{StN}$ & CRS (0.024") & Nomex Honeycomb & - & 79.25 & 179.76 & - & - \\
\hline
\end{tabular}


(d) Monolithic and sandwich materials

\begin{tabular}{|c|c|c|c|c|c|c|c|c|c|}
\hline \multirow[b]{2}{*}{ Material } & \multicolumn{2}{|c|}{ Density $\left(\mathrm{kg} / \mathrm{m}^{3}\right)$} & \multicolumn{2}{|c|}{ Young”s modulus (Pa) } & \multicolumn{2}{|c|}{ Poisson"s ratio } & \multicolumn{2}{|c|}{ Thickness (mm) } & \multirow[b]{2}{*}{ Mass (gram) } \\
\hline & Skin & Core & Skin & Core & Skin & Core & Skin & Core & \\
\hline CRS w/LE5208 & 7870 & 800.0 & $205 \mathrm{e} 9$ & $1.35 \mathrm{e} 9$ & 0.290 & 0.27 & 0.750 & 5.00 & 128.00 \\
\hline USIL Light & 7870 & 1990.0 & $205 \mathrm{e} 9$ & $12.85 \mathrm{e} 9$ & 0.290 & 0.26 & 0.375 & 0.75 & 38.20 \\
\hline Lexan & 1200 & & $2350 \mathrm{e} 9$ & & 0.375 & & 2.200 & & 21.00 \\
\hline
\end{tabular}

\section{NUMERICAL METHODS}

The Nastran and Abaqus Finite Element methods and the Matlab numerical solutions package were utilized for numerical solutions. Vibration eigensolutions were obtained via the Nastran procedure for circular plates, using the Hypermesh (version 10 from Altair Engineering Inc) pre- and post-processor for the selected frequency range 0 to $10,000 \mathrm{~Hz}$. A total of 9432 pshell elements were utilized with a triangular mesh of density 250 using the sol 103 (for normal modes) solution procedure. For the Abaqus procedure, all samples were also meshed by using the Hypermesh as a preprocessor and then Abaqus/Standard 3D was used as a solver to calculate the first three natural frequencies and the mode shapes of the samples. The Lanczos Eigensolver Method was selected in Abaqus/Standard 3D to determine the first three fundamental frequencies and mode shapes because this method is a powerful tool for extraction of the extreme eigenvalues and the corresponding eigenvectors of a sparse symmetric generalized eigenproblem. All samples were taken to be solid and the C3D4 (tetra 4) element type was used. For Matlab solutions, some reference code (Yang, 2005) was utilized in writing a Matlab computer program to calculate the natural frequencies and animate their mode shapes.

\section{RESULTS}

\subsection{Fabric Materials}

Figure 5 shows the acoustic absorption of fabrics The absorption coefficient in general increases monotonically with frequency. The thicker $\mathrm{B}$ and $\mathrm{K}$ fiberglass shows a much improved absorption over the thinner (9.5 versus $27 \mathrm{rmm}$, Table 2), other one.

\subsection{Foam Materials}

The absorption coefficient (Fig. 6) shows a number of maxima and minima. The polyurethane foam, which seems to perform best in the group, tested mainly has about 0.7 absorption, although this spikes to about 0.85 at about $6200 \mathrm{~Hz}$. The magic foams (two proprietary formulations) show resonance-type, rather than broadband behavior in their acoustic performances. They peak at multiple frequencies and manifest peaks that are for the major part, below the polyurethane level.

\subsection{Honeycomb Materials}

The acoustic responses of seven such structures are shown in Fig. 7a and basic vibration signatures in Fig. 7b. There is none of the materials that is a clearly better one over all the frequencies. The absorption coefficient curves manifest many peaks. The values generally increase until around 3-4 kHz, when they generally decrease. For a number of the materials, the highest absorption values approach unity.

\subsection{Monolithic and Sandwich Materials}

A material of one nature acting alone is here referred to as monolithic. Such materials may be used as skins for sandwiches and as partition materials. They tend to be metallic or other relatively acoustically hard materials. The monolithic material tested here is a single-layer lexan specimen and the two representative sandwich materials examined are Cold Rolled Steel (CRS)/LE5208, having cold rolled steel skins with a hard rubbery core LE5208 (proprietary product by L and L Inc) and also the USIL light with steel skins and thin polypropylene core.

Figure 8a and b show low acoustic absorption over a wide frequency range for these materials. The absorption of the CRS material is practically negligible at less than 0.1, while the USIL Light attains a low absorption of 0.15 to 0.2 in about a $200 \mathrm{~Hz}$ interval around $1100 \mathrm{~Hz}$. The monolithic solid lexan only achieves about 0.25 to 0.3 only in a narrow frequency band of about $1100 \mathrm{~Hz}$ to $1200 \mathrm{~Hz}$. Figure 8c shows the vibration modal spectrum of CRS/LE5208, charting the color-coded responses at the 17 impact hit points and consistently yielding the resonance frequencies.

Figure $7 \mathbf{b}$ is a histographic presentation of the first three resonance frequencies of the honeycomb samples. The CRS/nomex has the lowest resonances of all, while the honeycomb A (glass/epoxy with aluminum honeycomb core) has the highest. Figure 8d shows the contour-plot deformation shapes of the first three modes of vibration of the solid lexan sample, with a sample displacement legend. The results agree with all published literature. 
M. Al-Zubi et al. / American Journal of Applied Sciences 11 (10): 1784-1797, 2014

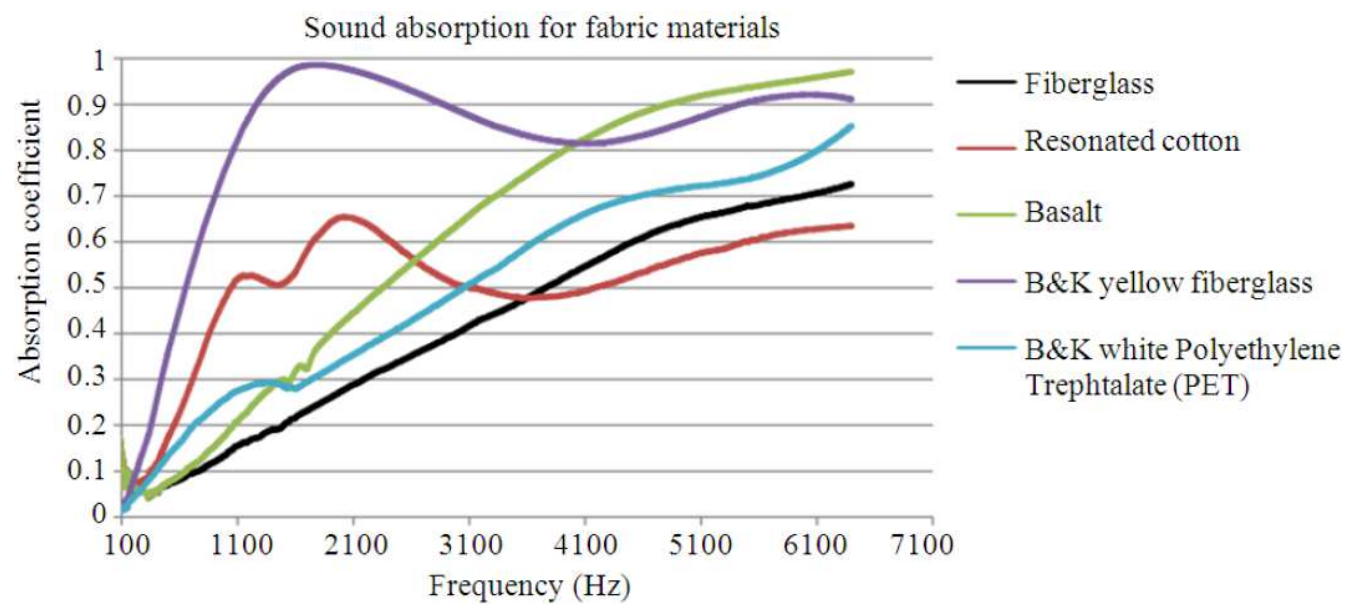

Fig. 5. Acoustic performance of fabric-type materials

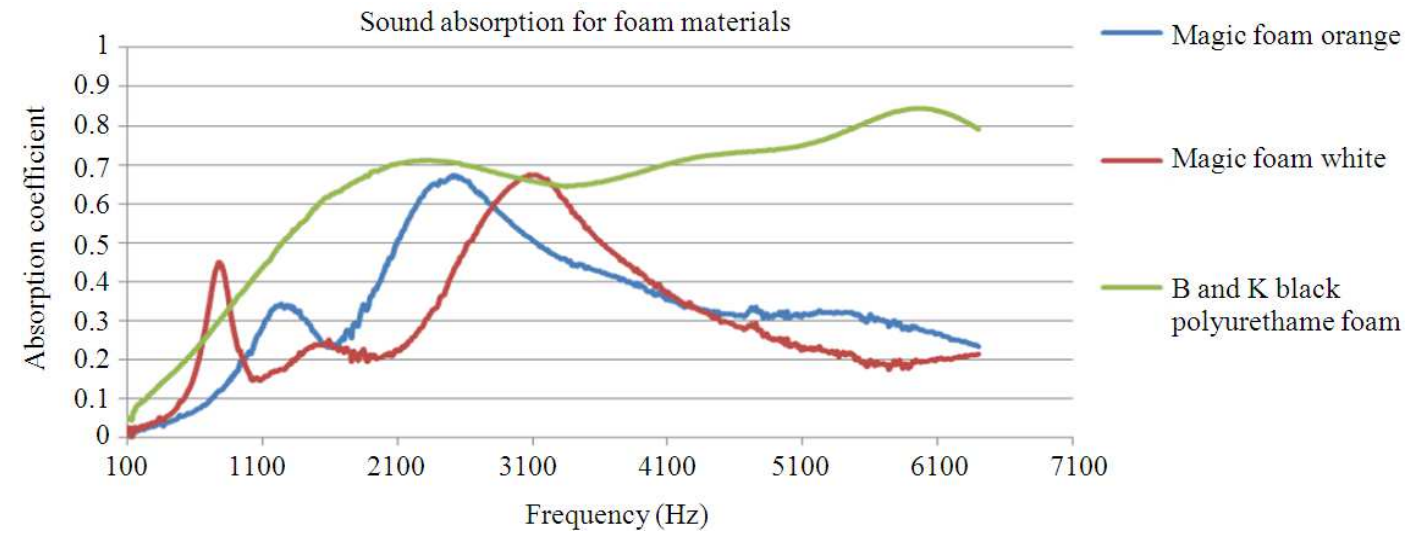

Fig. 6. Absorption coefficient versus frequency for foam materials

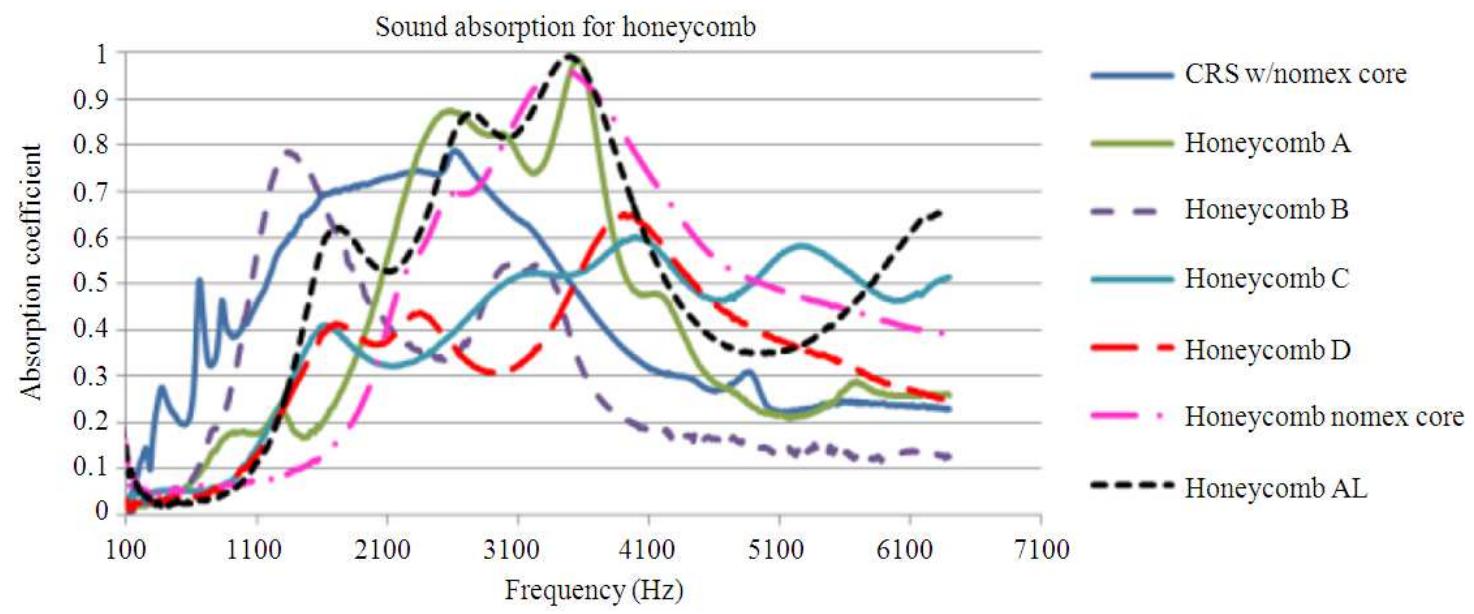

(a) 


\section{Comparison between Natural Frequencies}

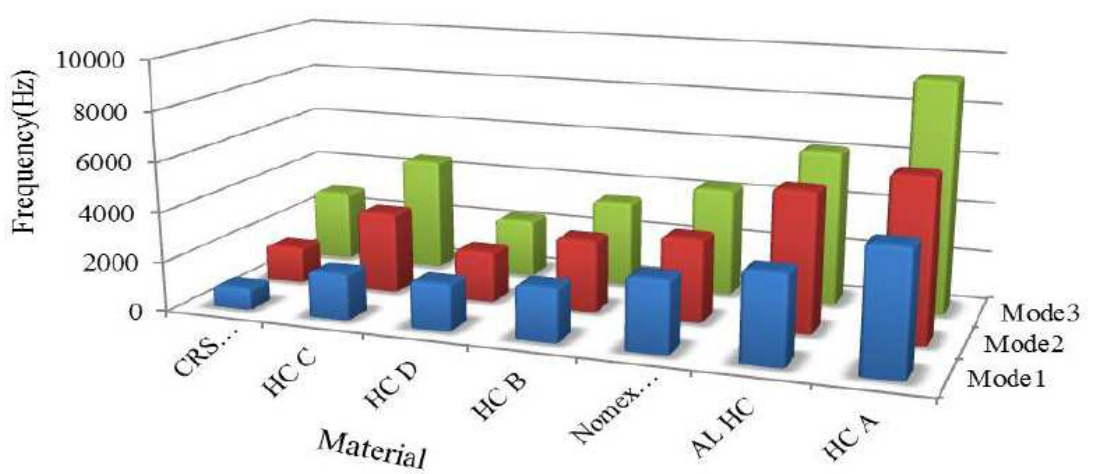

(b)

Fig. 7a. Absorption coefficient versus frequency for honeycomb, (b) Honeycombs vibration

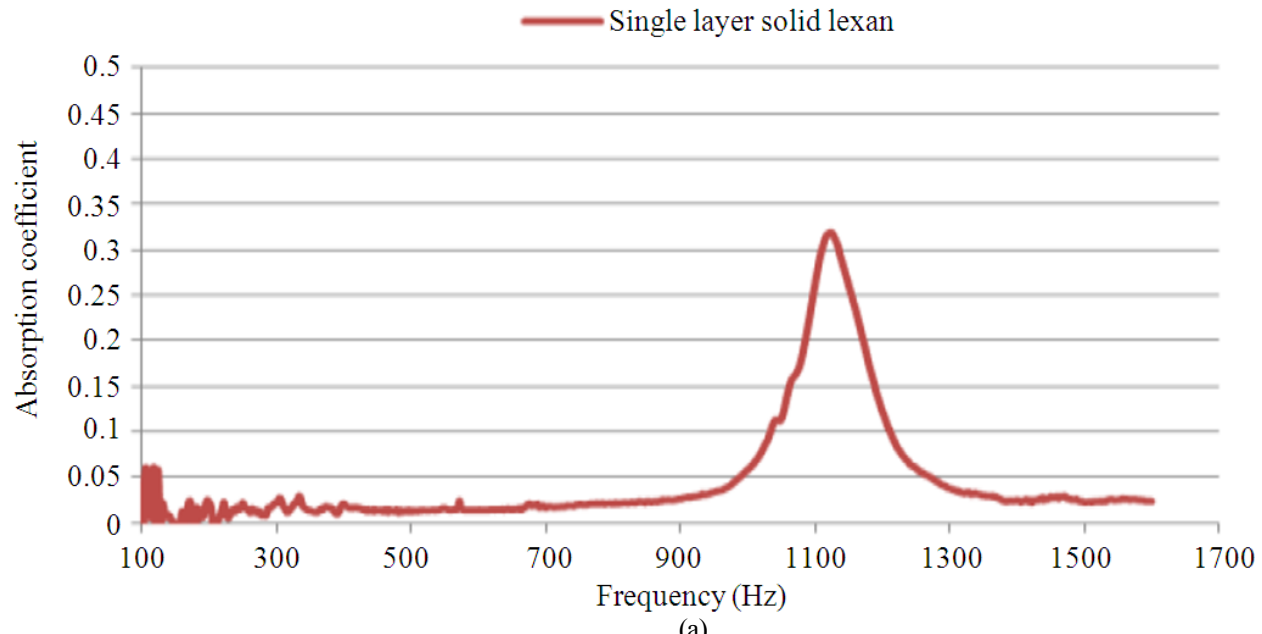

(a)

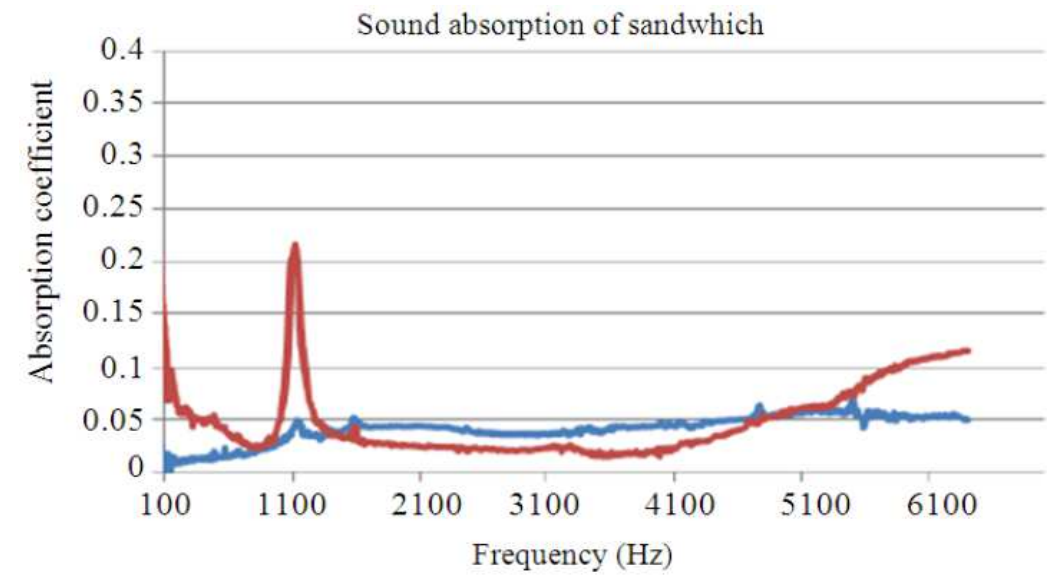

CRS w/LE 5208 USIL light

(b) 


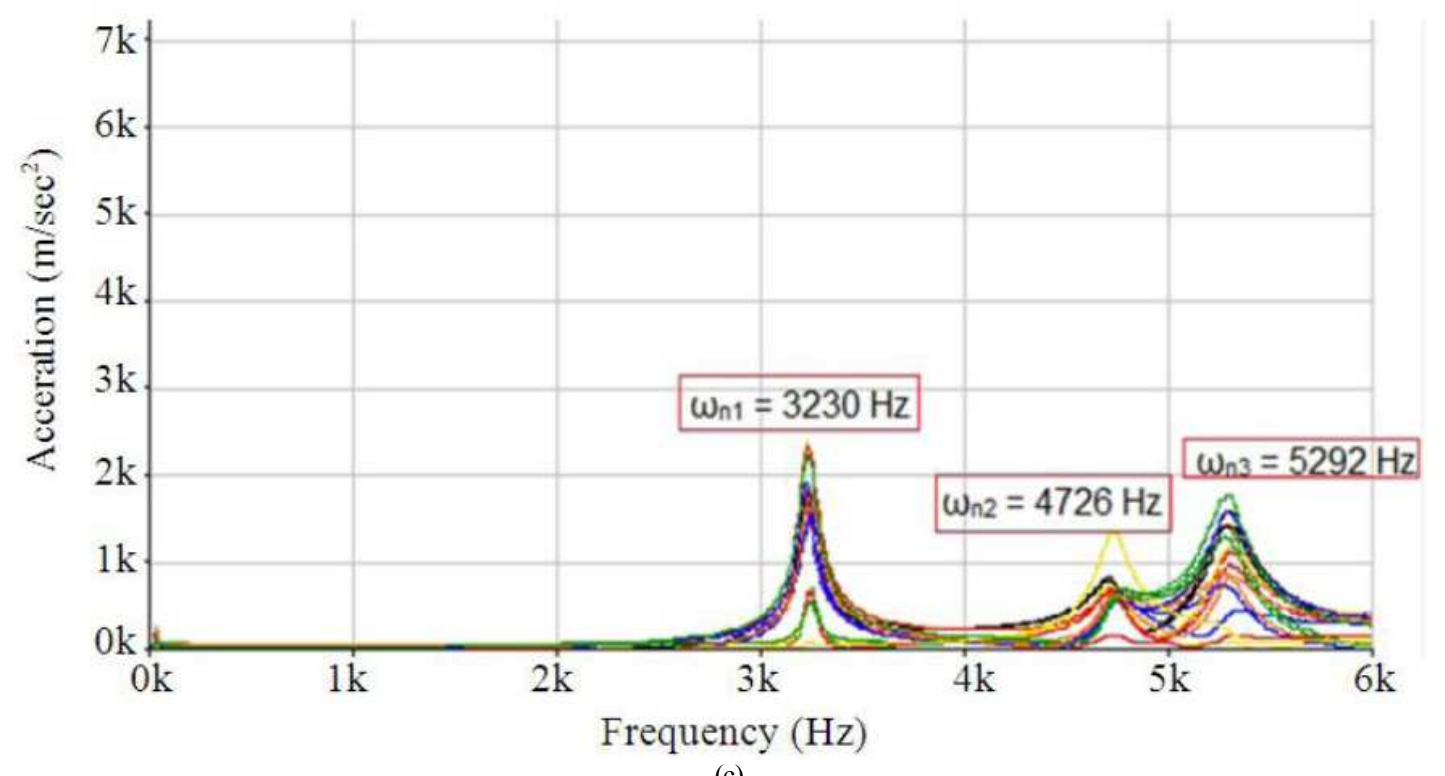

(c)
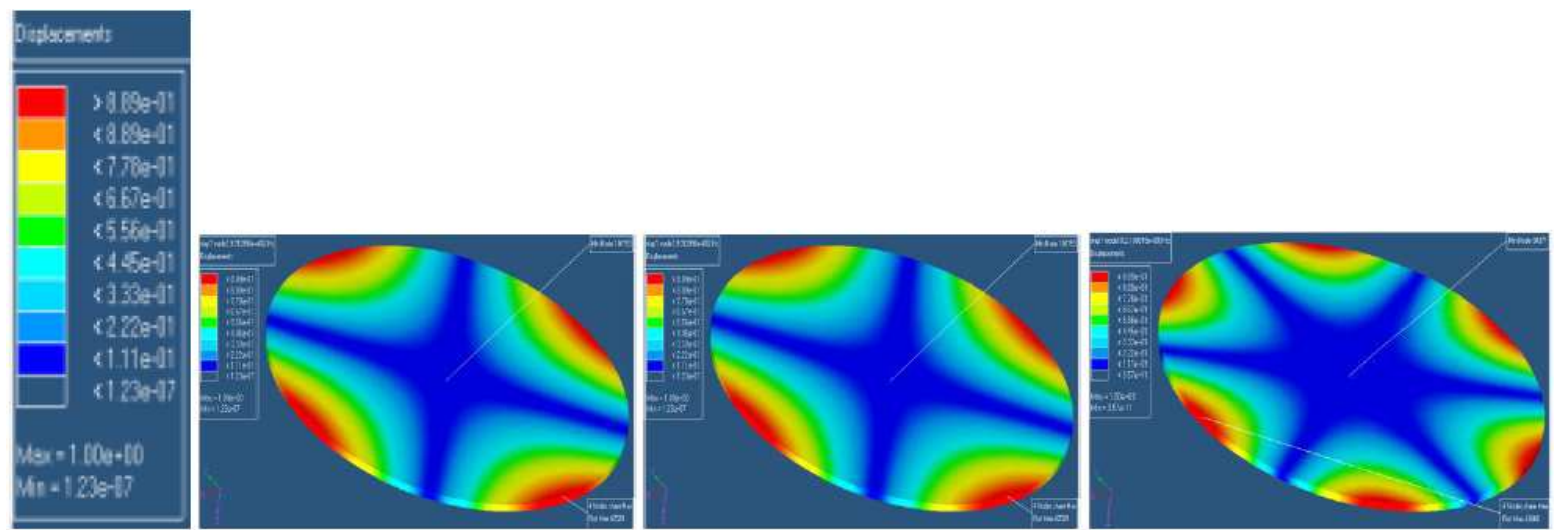

(d)

Fig. 8a. Absorption coefficient versus frequency for monolithic materials. (b) Absorption coefficient versus frequency for sandwich materials. (c) Vibration modal spectrum of CRS/LE 5208 sandwich. (d) Solid lexan-first 3 vibration modes

\section{DISCUSSION}

\subsection{Fabric Materials}

Figure 5 shows that the acoustic absorption of fabric materials is generally higher than 0.5 , from about $2000 \mathrm{~Hz}$. The yellow fiberglass and the resinated cotton actually cross the 0.5 absorption coefficient threshold at much earlier frequency values. The effect of higher density and wider thickness is to considerably increase acoustic absorption in the low frequency range and slightly decrease it in the higher frequency range. The low frequency waves have large wavelengths. For highly porous materials these waves can infiltrate the material for the absorptive action by viscous shear and thermal exchange, but cannot readily engage lowporosity materials. It may be concluded that basalt, fiberglass and PET show good absorption at high frequency values. This suggests that judicious combinations of materials may be made to most effectively target any frequency range for absorption increase. Fabric materials generally have high absorption coefficient at high frequencies and can effectively reduce high-frequency noise components. 
The thickness effect is also well illustrated in the results of Fig. 5. The thicker $\mathrm{B}$ and $\mathrm{K}$ fiberglass shows a much improved absorption over the thinner $(9.5 \mathrm{~mm}$ versus $27 \mathrm{~mm}$, Table 2) one. The increase of thickness is only beneficial up to a point, as sound pressure dies out with distance according to an exponential decrement law. The maximum useful thickness is often called the critical thickness. In practice, a thickness of about $40 \mathrm{~cm}$ is considered to be the maximum. Resinated cotton shows better performance than all other materials in the low frequency range (for similar thickness) because of its better ability to be set in motion by incident sound pressure than most other materials. This is why it has historically been widely applied for noise control.

Pieren et al. (2014) analyzed the acoustic behavior of fabrics with varying air flow resistances. His experimental and numerical results for three different types show that our results fall into the right zone.

\subsection{Foam Materials}

Foam materials are also widely applied for acoustic abatement on account of their lightness and efficiency in controlling sound of various frequencies. The absorption coefficient (Fig. 6) shows a number of peaks (max and $\mathrm{min}$ ) related to the thickness of the material. This maxmin profile is undesirable because of unsteadiness of the acoustic absorption value. In order to smooth out the absorption curve, random-sized wedge-shaped specimens do a better job. Acoustic response is dependent on foam cell behavior because the phenomena experienced with solid visco-elastic materials are also observed with porous materials that have been expanded from polymer stock. Thus, they are also subject to creep (strain increasing with time, for maintained stress), relaxation (stress decreasing with time for maintained strain), hysteresis (energy loss over each cycle, for cyclic loading) and strainrate dependence of effective stiffness/Young"s modulus. The propagation of both sound and vibration waves will be affected by the gas phase behavior, in particular the gas properties and entrapment pressure.

The overall acoustical behavior of the foam comes from the collection of the micro-structural characteristics of the local cells which themselves come from the frequency-dependent viscous and thermal parameters. The two key parameters are porosity and permeability. At low frequencies the viscous effect is dominant and at higher frequencies the viscous boundary layer becomes negligible and thermal effects dominate (the fluid moves as if it has no viscosity except at the boundary layer). Vibration induces pressure fluctuation in the fluid, which in turn induces temperature variation in the fluid-structure interface.

Foam materials (Fig. 6) appear to be not as good as fabric ones in containing sound. The performance of the magic foams is well below that of the polyurethane and is unsatisfactory. Some re-formulation of the magic foams seems to be required before they can give better acoustic response. It is proposed that this new class of magic foams be re-worked, especially as they are believed to have acoustic absorption characteristics that derive from their surface properties.

Ekici et al. (2012) investigated the acoustic response of foams, both neat and with augmenting materials like tea leaf fibers, and luffa-cylindrica fibers, for different thicknesses. Their findings confirm that the range of our absorption values are very realistic.

\subsection{Honeycomb Materials}

Honeycomb materials are used to combat excessive noise as they are light and contain ample air volumes that are useful in attenuating sound. Since they can be made to different geometries, a number of representative samples were examined for their acoustic behaviors.

The honeycomb structure is a typically complex one by virtue of the multiple units that are duplicated all over to make it up. Thus the occurrences of many internal resonances make its response to manifest several peaks, at varying frequencies. Figure 7a displays the acoustic responses of seven honeycomb structures. As mentioned before for foam materials, surface material placed on a porous or absorptive material acts in part as a springmass structure which tends to increase low-frequency absorption and decrease high-frequency absorption. Increasing thickness tends to have this effect as well in both porous and absorbent materials.

The nomex material was found to exhibit superior absorption for frequencies up to about $2000 \mathrm{~Hz}$. Thus, in the absence of other solutions, physical compounding of this material could be used with other types to extend the effective frequency range. Figure $7 \mathbf{b}$ shows the vibration behaviors of all honeycombs tested.

Acoustically, when a honeycomb structure is placed behind a porous absorbent layer it improves the absorption especially at low frequencies and can in fact broaden the frequency range of effectiveness. A very important property of the honeycomb is that its compartmental box structure forces the sound wave that intrudes into the honeycomb to propagate normal to the 
absorbing surface. The honeycomb structure has many resonant modes because of its box structure in which very many compartments may resonate locally. Hence, the acoustic absorption and Frequency Response Function (FRF) curves may show multiple peaks.

The honeycomb materials were tested for vibration behavior using PULSE 16.1. To verify the experimental results, finite element simulation was used to get the natural frequencies and mode shapes for each of the samples. The analysis of the vibration and acoustics of honeycomb materials involves at least four key stiffness and strength properties in each direction-longitudinal and transverse. These properties are Young"s modulus, shear modulus, tensile strength and compressive strength. The failure modes of the honeycomb are usually local crushing, bending fracture, compression fracture, global buckling, face wrinkling with debonding, face wrinkling with core-crushing and shear crimping.

The work of Lin et al. (2010) considered five thicknesses of various combinations of PET honeycomb and low-melting temperature plastics. It can be seen from their absorption coefficient curves that our values for various honeycomb structures tested are appropriate.

\subsection{Monolithic and Sandwich Material}

The absorption coefficients of these materials, as shown in Figs. 8a and b, are so low because the materials are acoustically hard. Generally, the rate at which a flexible panel absorbs acoustic energy is proportional to the product of the amplitude and frequency of vibration, internal damping and frictional losses at its mounting edges. Fig. 8b shows very low acoustic absorption over a wide frequency range for the CRS sandwich material, due to its core being very hard. However, over a small frequency band, there is an improved, but still modest absorption for the USIL light. LE5208 is a hard, closed-cell foam that resists effective intrusion of sound-bearing air into it. USIL light is much thinner than the CRS $(1.5 \mathrm{~mm}$ versus $6.5 \mathrm{~mm}$ total thickness) and is thus more vibrated by sinusoidal pressure than CRS.Such materials as these might be used for their vibration properties, but contribute relatively little to acoustic performance.

Lexan (a polycarbonate plastic) was examined as a monolithic materials. The low values of lexan coefficient in the range (Fig. 8a) show that this material is not good for containing sound. Figure 8d indicates that the lexan material shows distinctness or clarity of vibration modes. Figure 8c essentially collates the vibration responses obtained from the 17 impact test hit points for the CRS/LE5208 sandwich. This is an example, as the tests were done for all materials. Individual variations occur among the results from hitting different points because of the relative nearness or otherwise to the particular hit point of nodal lines in a given vibration mode. The fact that the resonance frequency values in this figure are high, which is desirable in vehicle applications as they are thus higher than the major troublesome system and sub-system resonances and thus cannot interact with these to cause destructively high deformations and consequential stresses.

Frommhold et al. (1992) investigated the acoustic absorption of a metal membrane box cavity draped around with a thinner metal membrane and variously with differ ent hole dimeters or none. The acoustic absorption of the holeless metal construction compares with our results, altough perforations, which essentially add notable helmholtz resonator absorption effects appreciably enhance performance.

\section{CONCLUSION}

The experiments, analyses and numerical work in this study have enabled the systematic examination of various types of materials and architectures for suitability for NVH containment. The results have shown that fibrous and fabric-type materials tend to possess high acoustic absorption capabilities. Thus they could be utilized to form part of synthesized panel materials to augment acoustic capability.

As seen in Fig. 7 the acoustic absorption coefficients of hard-core, hard-layered materials tend to be low, unless the cores contain high-absorption materials. The acoustic absorption capabilities of traditional honeycomb, materials were found to be high in the mid-frequency range (about 1500 to $4500 \mathrm{~Hz}$ ). This is believed to be mainly due to the fact that the air contained in the cells is sealed in and this enables the incident sound to be destructively reflected multiples of times. The acoustic absorption coefficients of nontraditional-honeycomb, Periodic Cellular Material Structures (PCMS) had been found (Al-Zubi et al., 2013) to be generally low wherever the in-structure air pockets were not confined and hence the acoustic damping of air was ineffective. In practice such materials will have to be augmented with highabsorption materials as fillers and/or face attachments for better acoustic performance.

With respect to vibration response, considering in general the first few modes, maximum vibration 
amplitude generally decreased with increase in mode number, although for more complicated structures deviations to this occurred because of the presence of many sub-systems in such structures. As expected, vibration amplitude at the same mode was observed to depend on location of hitting point. This would be due to the relative distance of such an impact point from, or proximity to a nodal line. The modal analysis results (collected over all 17 hit points) seem to point out the resonant frequencies more easily and more accurately.

\section{REFERENCES}

Allard, J.F., C. Depollier, P. Guignouard and P. Rebillard, 1999. Effect of a resonance of the frame on the surface impedance of glass wool of high density and stiffness. J. Acoustical Soc. Am., 89: 999-1001. DOI: 10.1121/1.400642

Al-Zubi, M.A., E.O. Ayorinde, M.A. Dundar, M. Warriach and Y. Murty, 2013. Vibro-acoustic characterization and optimization of Periodic Cellular Material Structures (PCMS) for NVH applications. J. Mater. Sci. Res. DOI: 10.5539/jmsr.v2n4p64

ASI, 2012. Chaska, MN, Soundproofing, acoustics, noise and vibration control specialists. Echo eliminatorBonded acoustical cotton product data. Acoustical Surfaces Inc.

Biot, M.A., 1956. Theory of propagation of elastic waves in a fluid-saturated porous solid. 1. Low-frequency range. J. Acoustical Society Am., 28: 168-178. DOI: 10.1121/1.1908239

Dym, C.L. and M.A. Lang, 1974. Transmission of sound through sandwich panels. J. Acoustical Soc. Am., 56: 1523-32. DOI: 10.1121/1.1903474

Ekici, B., A. Kentli and H. Kutchuk, 2012. Improving sound absorption property of polyurethane foams by adding tea-leaf fibers. Archives Acoustics, 37: 515520. DOI: $10.2478 / \mathrm{v} 10168-012-0052-1$

Frommhold, W., H.V. Fuchs, S. Sheng, 1992. Acoustic performance of membrane absorbers. J. Sound Vibration, 170: 621-636. DOI: 10.1006/jsvi.1994.1091

Ho, J.H. and A. Berkhoff, 2014. Comparison of various decentralised structural and cavedback control strategies for transmitted noise reduction through a double panel structure. J. Sound Vibration, 333: 1857-1873. DOI: 10.1016/j.jsv.2013.11.018
Hoshino, H., T. Sakurai and K. Takahashi, 2003. Vibration reduction in the cabins of heavy-duty trucks using the theory of load transfer paths. JSAE Rev., 24: 165-171. DOI: 10.1016/S03894304(03)00005-5

Jain, D., 2002. Modeling the characteristics of newgeneration ford vehicle panels for efficient noise, vibration and harshness performance. MSc., Thesis, Wayne State University.

Kirchhoff, G., 1850. Llber das Gleichgewicht und die Bewegung einer elastischer. Scheibe. J. Filr Math.

Kurtze, G., 1959. Bending wave propagation in multilayer plates. Acoustical Soci. Am. J., 31: 1183-1201. DOI: 10.1121/1.1907848

Lagrange, J.L., 1815. Mécanique Analytique. 2nd Edn., Harvard University, Paris, Ve Courcier, pp: 378.

Lin, J.H., C.M. Lin, C.C. Huang, C.C. Lin, and Y.C. Liao et al., 2010. Evaluation of the manufacture of sound absorbent sandwich plank made of PET/TPU honeycomb grid/PU foam. J. Compt. Matls., 45: 1355-1362. DOI: $10.1177 / 0021998310381438$

Parry-Jones, R. and J.M. Weaver, 1999. Design for NVH University of Detroit-Mercy.

Poisson, S.D., 1894. Memoir sur $1^{\text {ee }}$ equibre et le movement des corp elastique. M, Em Acad.

Pieren, R., K. Heutschi, M. Müller, M. Manyoky and K. Eggenschwiler, 2014. Auralization of wind turbine Noise: Emission synthesis. Acta Acustica United Acustica, 100: 25-33. DOI: 10.3813/AAA.918683

Rayleigh, J.W.S.B., 1945. The Theory of Sound. 1st Edn., Courier Dover Publications, Magnolia, Mass, ISBN-10: 0486602923, pp: 520.

Ross, A., 2006. Basalt fibers: Alternative to glass. Composites Technol., 12: 44-48.

Timoshenko, S., 2011. Vibration Problems in Engineering. 2nd Edn., Oxford City Press, New York, ISBN-10: 1849025134, pp: 480.

Vardan, H., 2003. Vibro-acoustics now key to automotive competition. Noise Vibrat. Worldwide, 34: 13-15. DOI: 10.1260/09574560360698057

Wang, B., S. Zheng, L. Zhou, S. Liu and X. Lian et al., 2010. Acoustic modelling and analysis of vehicle interior noise based on numerical calculation. Proceedings of the International Conference on Intelligent Computation Technology and Automation, May 11-12, IEEE Xplore Press, Changsha, pp: 404407. DOI: 10.1109/ICICTA.2010.582 
Wang, W., J.E. Mottershead and C. Mares, 2009. Vibration mode shape recognition using image processing. J. Sound Vibrat., 326: 909-938. DOI: 10.1016/j.jsv.2009.05.024

Woodcock, R. and J. Nicolas, 1995. A generalized model for predicting the sound transmission properties of generally orthotropic plates with arbitrary boundary conditions. J. Acoust. Soc. Am. DOI: 10.1121/1.412222
Yang, B., 2005. Stress, Strain and Structural Dynamics: An Interactive Handbook of Formulas, Solutions and MATLAB Toolboxes. 1st Edn., Academic Press, Amsterdam, Boston, Mass, ISBN-10: 0127877673, pp: 942.

Zwikker, C. and C.W. Kosten, 1949. Sound Absorbing Materials. 1st Edn., Elsevier Pub. Co., New York, pp: 174. 